15

\title{
Разработка системы напуска газа в ионный источник инжектора атомов токамака Глобус-М и оптимизация с ее помощью параметров атомного пучка
}

\author{
(C) А.Ю. Тельнова, ${ }^{1}$ В.Б. Минаев, ${ }^{1}$ П.Б. Щеголев, ${ }^{1}$ Д.В. Разуменко, ${ }^{1}$ Г.С. Курскиев, ${ }^{1}$ А.В. Лупин ${ }^{2}$ \\ ${ }^{1}$ Физико-технический институт им. А.Ф. Иоффре, \\ 194021 Санкт-Петербург, Россия \\ ${ }^{2}$ Санкт-Петербургский политехнический университет Петра Великого, \\ 195251 Санкт-Петербург, Россия, \\ e-mail: Anna.Telnova@mail.ioffe.ru
}

(Поступило в Редакцию 16 июля 2016 г.)

Разработана новая система напуска газа в газоразрядную камеру источника ионов, обеспечивающая возможность регулировать режим горения дугового разряда и параметры плазменного эмиттера ионов. Выполнены измерения основных электротехнических параметров ионного источника, сигналов с вторичноэмиссионных зондов. На основе полученных данных восстановлен профиль распределения мощности в пучке. Подобрана оптимальная форма сигнала управления клапаном газонапуска инжектора атомов сферического токамака Глобус-М.

DOI: 10.21883/JTF.2017.07.44685.1959

\section{Введение}

Комплекс нейтральной инжекции [1] был впервые задействован в плазменном эксперименте на сферическом токамаке Глобус-М [2] в конце 2002 г. С тех пор нейтральная инжекция стала одним из основных методов дополнительного нагрева плазмы на установке.

Первоначально оборудование комплекса, разработанное в 70-е годы в НИЦ „Курчатовский институт“ для токамака T-11, после переезда в ФТИ им. А.Ф. Иоффе было введено в эксплуатацию с минимальными доработками, необходимыми для согласования с остальными системами токамака. Была обеспечена возможность инжекции водородного или дейтериевого пучка с энергией частиц до $30 \mathrm{keV}$ в течение $30 \mathrm{~ms}$. Максимальная мощность нейтрального пучка на выходном патрубке инжектора составила 0.5 или $1.0 \mathrm{MW}$ в зависимости от типа установленного источника ионов (ИПМ-2 или ИПМ-1 соответственно). Плазменный эмиттер ионов формировался в газоразрядной камере (ГРК) с помощью дугового разряда. Напуск рабочего газа в камеру осуществлялся с помощью электромагнитного клапана, a его поток регулировался между выстрелами вручную с помощью встроенного игольчатого натекателя. Перезарядочная мишень для ускоренного пучка ионов формировалась в нейтрализаторе из газа, вытекающего из ионного источника.

С целью расширения экспериментальных возможностей установки была доработана система питания инжектора и увеличена длительность импульса инжекции свыше $50 \mathrm{~ms}$. Как показали измерения, существовавшая система напуска газа в разрядную камеру источника, работавшая в „ключевом“ режиме, не обеспечивала оптимальные параметры плазменного эмиттера в течение всего увеличенного по длительности импульса инжекции. В частности, это сказывалось на ухудшении фокусировки пучка по вертикали из-за отклонения от оптимального значения величины вытягиваемого из источника тока во второй половине импульса. В качестве решения было предложено регулировать эмиссию ионов, изменяя во времени поток рабочего газа, поступающего в ГРК источника. Кандидатом на роль регулирующего напуск газа устройства был выбран пьезоэлектрический клапан. Его пропускная способность определяется приложенным напряжением, что позволяет оперативно управлять режимом горения дугового разряда в ГРК источника ионов и оптимизировать параметры плазменного эмиттера. Сложность использования такого клапана на инжекторе обусловлена необходимостью его размещения в непосредственной близости от ГРК под высоким потенциалом (до $30 \mathrm{kV}$ ) и чувствительностью

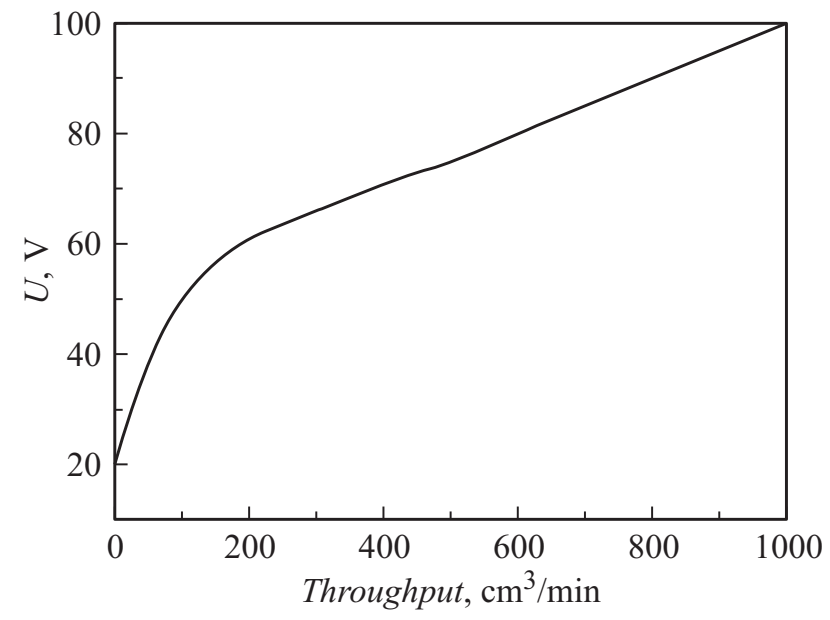

Pис. 1. Пропускная способность клапана MaxTek MV112 в зависимости от приложенного к нему напряжения. 

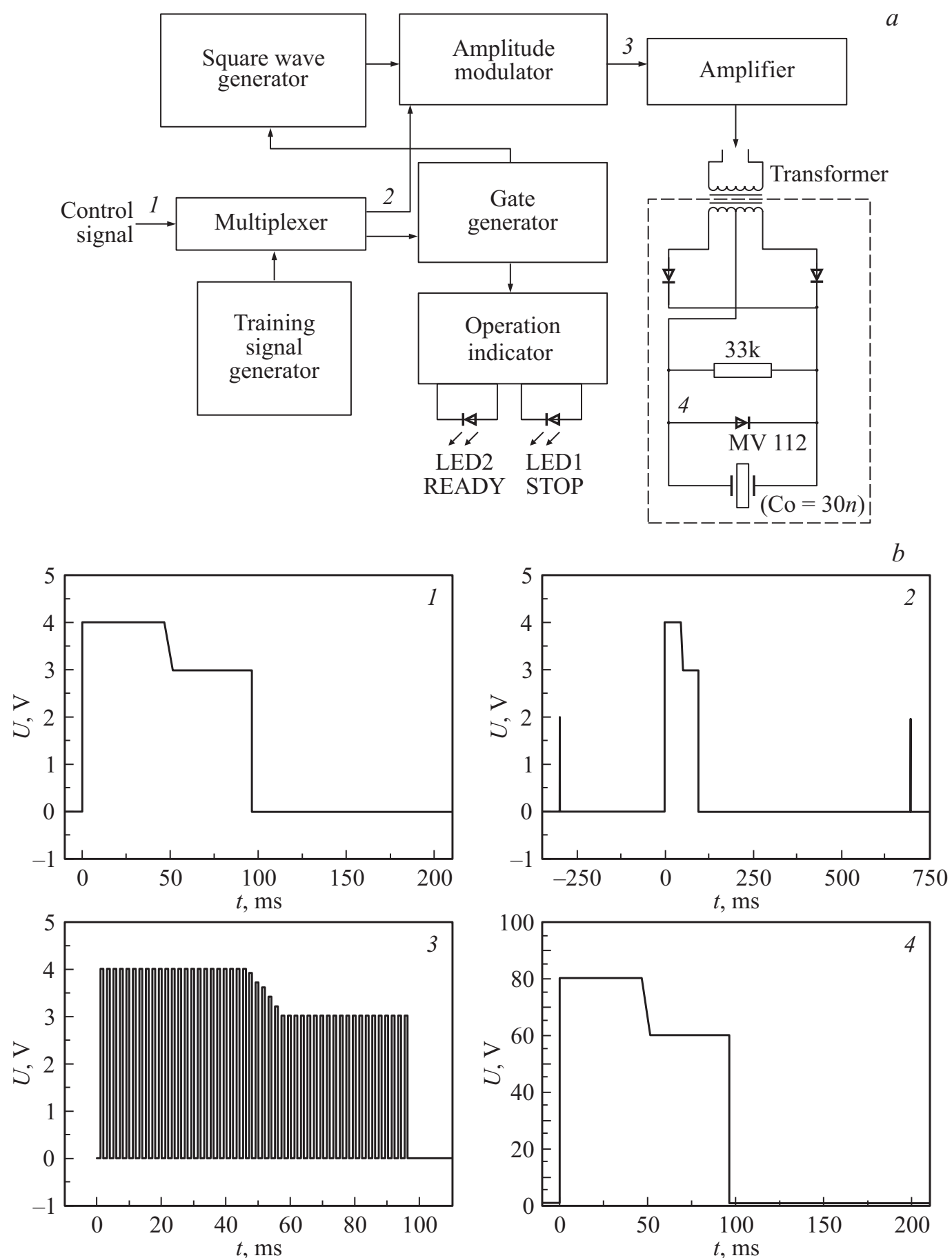

Рис. 2. Модуль защиты и управления пьезоэлектрическим клапаном: $a-$ функциональная блок-схема; $b-$ электрические сигналы в контрольных точках. Control signal - управляющий сигнал, Training signals generator - генератор тренировочных сигналов, Multiplexer - мультиплексор, Operation indicator - индикатор работы, Gate generator — генератор „ворот“, Amplitude modulator - амплитудный модулятор, Square wave generator - генератор меандра, Amplifier — усилитель, Transformer — трансформатор.

пьезоэлектрического элемента к электромагнитным наводкам, возникающим при высоковольтных пробоях в ускоряющей пучок системе. Настоящая работа посвящена разработке и испытаниям новой системы напуска рабочего газа в ГРК ионного источника инжектора токамака Глобус-М.

\section{1. Система напуска газа}

В качестве устройства, регулирующего поток газа в новой системе газонапуска инжектора атомов, был выбран клапан MaxTek MV112 [3]. Его пропускная способность в зависимости от приложенного к нему 
Таблица 1. Основные характеристики АЦП/ЦАП Е-14-140MD

\begin{tabular}{c|c}
\hline \multicolumn{2}{|c}{ АЦП } \\
\hline Количество каналов & 16 дифференциальных или 32 с „общей землей“ \\
\hline Разрядность АЦП & 14 bit \\
\hline Количество каналов & ЦАП \\
\hline Разрядность & 16 bit \\
\hline Синхронизация & Возможна многомодульная синхронизация \\
\hline Выходной диапазон & $\pm 5 \mathrm{~V}$ \\
\hline Выходной ток, не более & $\pm 10 \mathrm{~mA}$ \\
\hline Максимальная частота выдачи данных & $200 \mathrm{kHz}$ \\
\hline
\end{tabular}

Питание - от шины USB

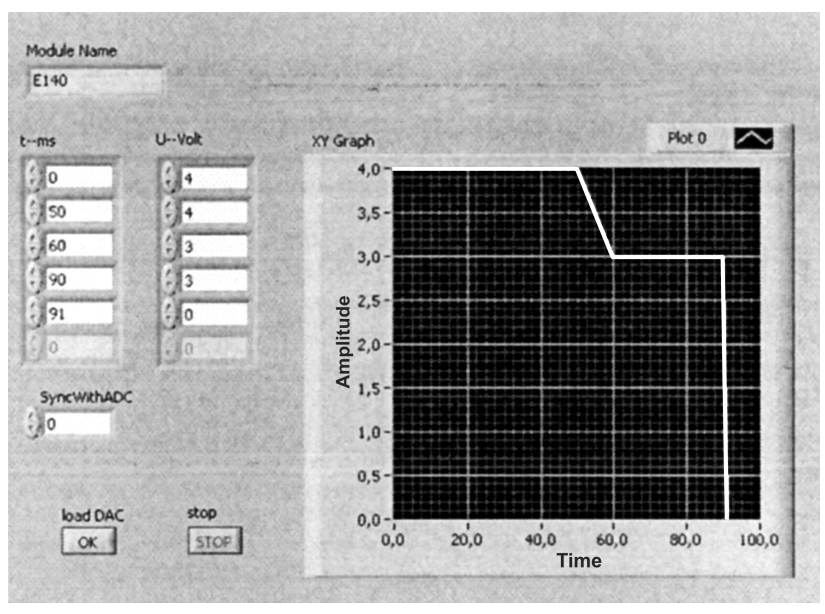

Рис. 3. Интерфейс программы управления клапаном.

напряжения приведена на рис. 1. Диапазон входных напряжений составляет $0-100 \mathrm{~V}$.

Одним из недостатков данного клапана является сильная нелинейность зависимости пропускной способности от приложенного напряжения на начальном участке, проявляющаяся как эффект „залипания“ и снижающая стабильность срабатывания при приходе управляющего импульса. Для устранения указанного недостатка было предложено непрерывно подавать на клапан импульсы, которые эффективно устраняют эффект залипания, но при этом не открывают сам клапан.

При создании новой системы газонапуска было выполнено конструирование тракта подачи газа, разработан специальный модуль защиты и управления пьезоэлектрическим клапаном, создан программный интерфейс для управления клапаном с ПК.
Одним из основных требований при конструировании системы подачи газа было максимально близкое расположение регулирующего элемента к объему ГРК для повышения быстродействия системы. В разработанной конструкции клапан расположен на расстоянии $150 \mathrm{~mm}$ от ГРК и соединен с ней с помощью трубки из нержавеющей стали с внутренним диаметром $4 \mathrm{~mm}$. Для гальванической развязки ресивера с рабочим газом от клапана, находящегося под потенциалом эмиссионного электрода, была использована фторопластовая трубка. Сам клапан был помещен в алюминиевый корпус, для экранирования от внешних наводок.

В качестве устройства формирования управляющего сигнала был использован стандартный внешний модуль фирмы L-card E-14-140MD [4], связанный с управляющим компьютером через USB-интерфейс. Основные характеристики модуля приведены в табл. 1. Формирование импульса осуществляется программно или по приходу внешнего запускающего импульса.

Сформированный аналоговый сигнал управления по коаксиальному кабелю $(\sim 30 \mathrm{~m})$ передается в экспериментальный зал на специально разработанное устройство - модуль защиты и управления пьезоэлектрическим клапаном [5]. Он предназначен для согласования выходного сигнала задающего устройства (ЦАП) с входным сигналом пьезоклапана по уровню; для передачи сигнала от задающего устройства, находящегося под потенциалом земли, к пьезоклапану, находящемуся под потенциалом эмиссионного электрода $\left(U_{e e}\right)$; и для защиты клапана от случайных срабатываний при пробое в ионном источнике. Функциональная схема устройства и сигналы в основных контрольных точках представлены на рис. 2.

Тренировочные импульсы (частотой $1 \mathrm{~Hz}$, длительностью $100 \mu \mathrm{s}$, амплитудой $2 \mathrm{~V}$ ), предотвращающие залипание, вырабатываются самим устройством и подаются на 

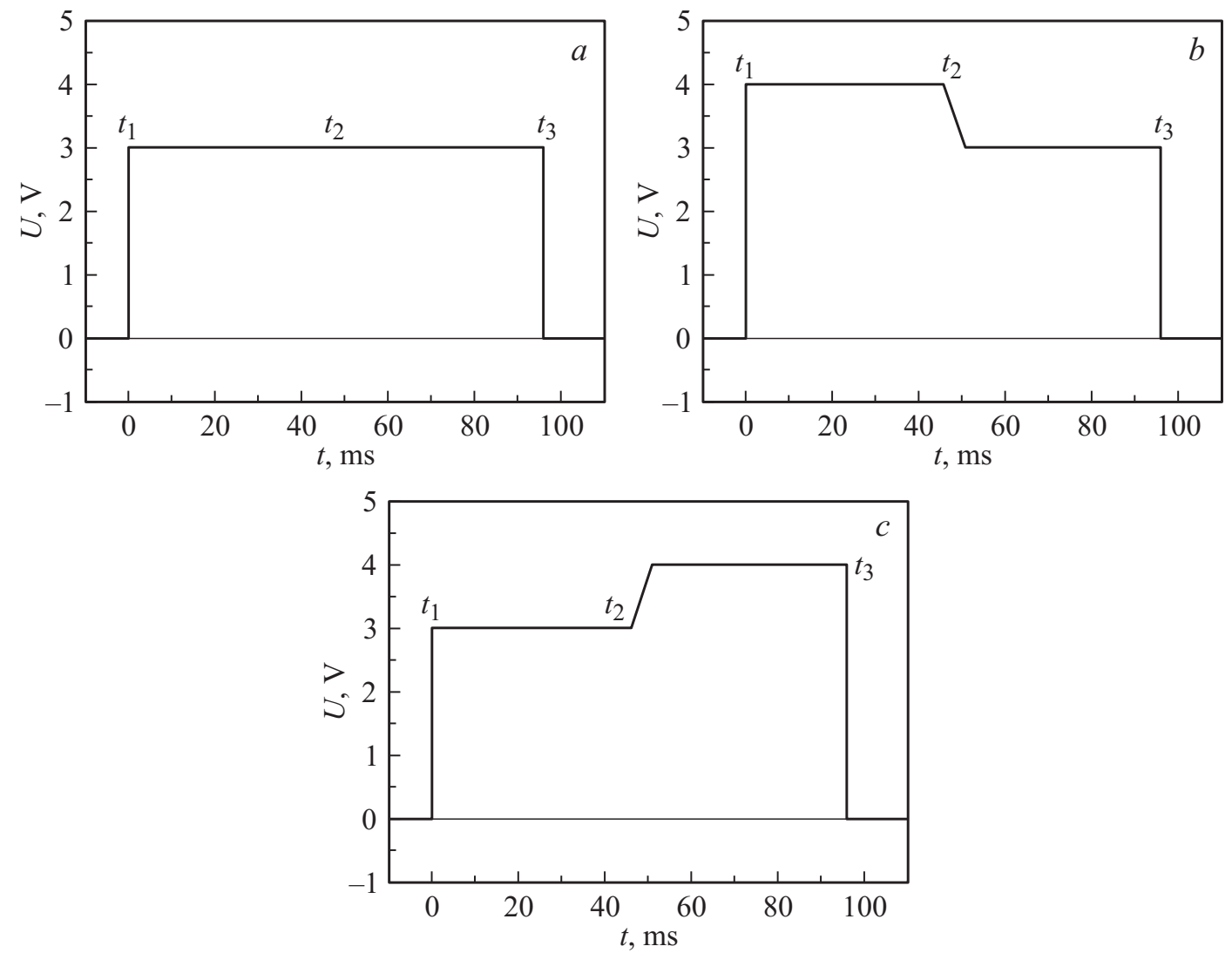

Рис. 4. Форма управляющих импульсов, подававшихся на клапан в разрядах \# 320686 (a), \#320710 (b) и \# 320693 (c).

Таблица 2. Параметры работы инжектора во время проведения испытаний

\begin{tabular}{l|c}
\hline Ионный источник & ИПМ-2 \\
\hline Рабочий газ & Водород \\
\hline Напряжение эмиссионного электрода & $23 \mathrm{kV}$ \\
\hline Длительность дугового разряда & $50 \mathrm{~ms}$
\end{tabular}

пьезоклапан непрерывно между выстрелами инжектора. В момент прихода на мультиплексор управляющего сигнала 1 передача тренировочных импульсов на клапан прекращается. Мультиплексный сигнал 2 поступает на генератор „ворот“ и амплитудный модулятор. Генератор ворот задает максимальное время нахождения пъезоклапана в открытом состоянии, ограничивая длительность пропускаемого на него импульса $200 \mathrm{~ms}$. Сигнал генератора ворот разрешает работу генератора меандра, генерирующего сигнал с частотой $100 \mathrm{kHz}$. Этот сигнал поступает на амплитудный модулятор, где происходит его модуляция управляющим сигналом. Далее сигнал преобразуется в двуполярный и усиливается до амплитуды $\pm 50 \mathrm{~V}$. По коаксиальному кабелю $(\sim 13 \mathrm{~m})$ усиленный сигнал передается на трансформатор, обеспечивающий высоковольтную развязку. Со вторичных обмоток трансформатора сигнал поступает на схему двуполупериодного выпрямителя. Роль конденсатора сглаживающего фильтра выполняет пьезоэлемент клапана, обладающий емкостью около $30 \mathrm{n}$. Максимальное значение управляющего напряжения на пьезоклапане составляет $100 \mathrm{~V}$ и его амплитуда ограничена с помощью стабилитрона.

Форма, амплитуда и длительность управляющего сигнала, также как и режим работы устройства, задаются в промежутках между выстрелами инжектора через специально разработанный программный интерфейс (рис. 3). В окне „Module name“ выводится модель подключенного модуля, генерирующего управляющий сигнал. В представленном на рисунке случае - это модуль E-14-140MD фирмы „L-card“. C помощью кнопки „Load DAC“ осуществляется генерация одного управляющего импульса в ручном режиме. Кнопка „Stop“ позволяет прервать генерацию управляющего сигнала. Окно „SyncWithADC“ определяет режим синхронизации модуля с инжектором. При значении параметра, равном „, $0^{“}$, синхронизация отключена, и открытие клапана производится вручную нажатием кнопки „Load DAC“. При значении, равном „1“, клапан открывается по внешнему импульсу, поступающему от синхронизатора инжектора. С помощью окон колонок „t-ms“ (время) и „U-Volt“ (амплитуда) задается форма управляющего импульса, которая отображается на графике справа. 

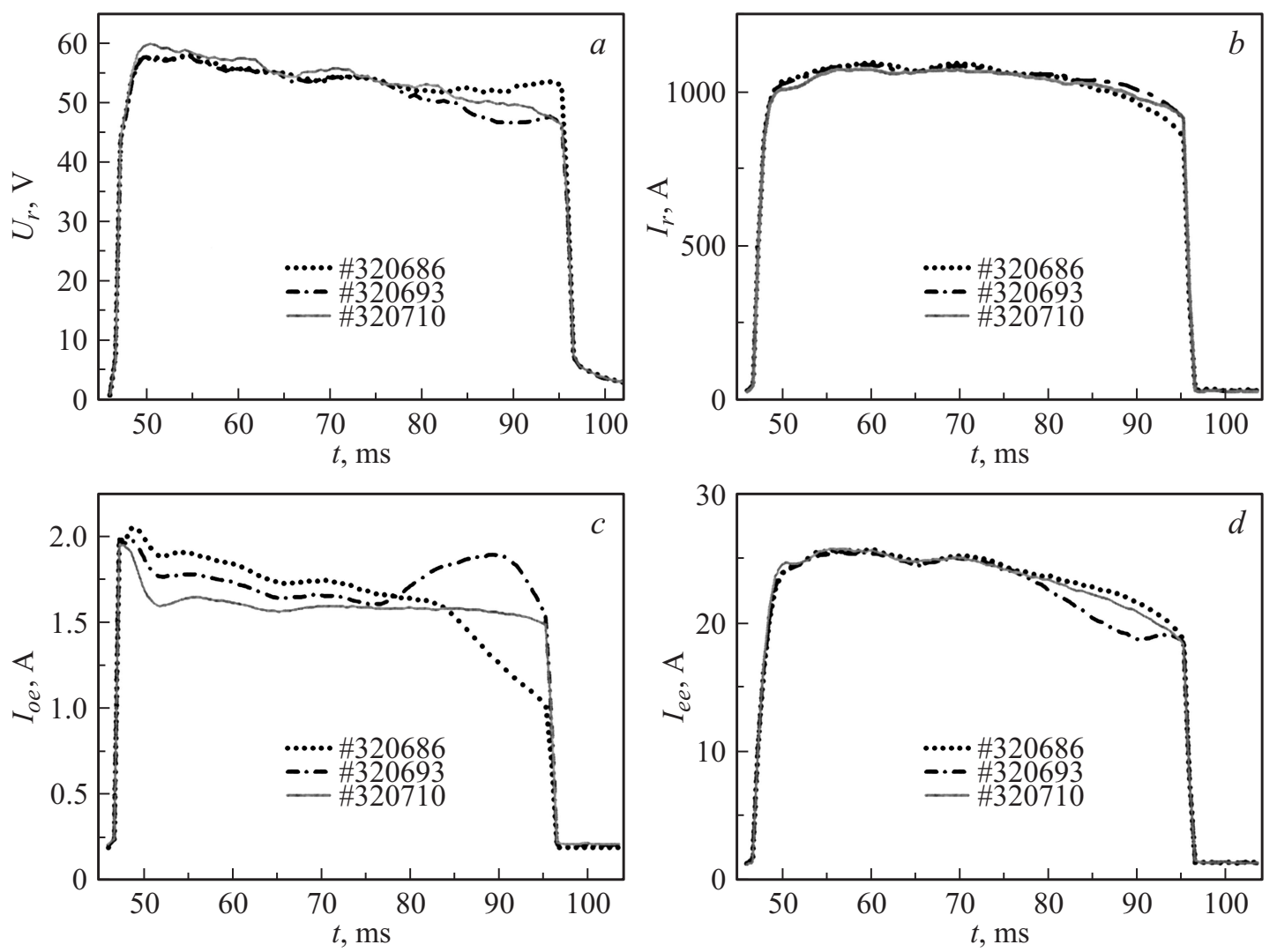

Рис. 5. Осциллограммы напряжения разряда $(a)$, тока разряда электрода $(d)$ для разрядов \# 320686, \# 320710, \# 32069.

\section{2. Испытания системы напуска газа на инжекторе и оптимизация параметров пучка}

Для испытаний новая система напуска газа была установлена на источник ионов ИПМ-2 взамен старой. В ходе эксперимента исследовалось влияние режима напуска рабочего газа в ГРК на параметры дугового разряда и эмиссионные характеристики источника, а также эффективность нейтрализации ионов. Параметры работы инжектора во время испытаний приведены в табл. 2.

Во время испытаний на клапан подавались управляющие сигналы различной формы. Всего было проработано порядка 30 вариантов управляющих сигналов. Ниже приведены три из них (рис. 4). Время $t_{1}$ соответствует моменту прихода синхроимпульса от синхронизатора инжектора и началу формирования газовой мишени перезарядки, $t_{2}$ - поджигу дугового разряда в ионном источнике, $t_{3}$ - окончанию газонапуска и закрытию клапана перед окончанием разряда.

Влияние изменения режима напуска газа в ГРК на напряжение $U_{r}$ и ток разряда $I_{r}$, а также на токи эмиссионного $I_{e e}$ и отрицательного $I_{o e}$ электродов представлено на рис. 5. Видно, что разработанная схема позволяет регулировать в течение импульса напуск газа в разряд и тем самым влиять на его характеристики и

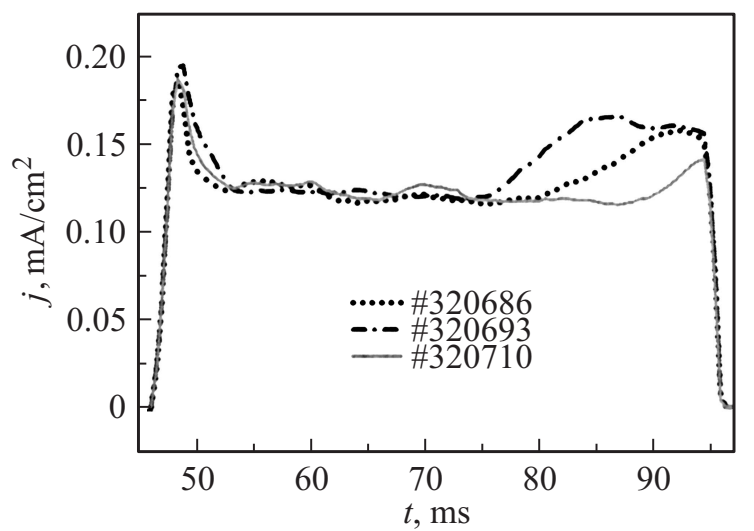

Рис. 6. Токовые сигналы с вторично-эмиссионного зонда для разрядов \# 320686, \# 320710, \# 320693 с различными режимами напуска газа в ГРК.

режим работы ионного источника в целом. При этом наибольшее постоянство ток эмиссионного электрода демонстрирует на протяжении разряда \# 320710. Следует заметить, что при неизменном режиме напуска газа наблюдается хорошая повторяемость всех сигналов от разряда к разряду.

Выбор оптимального режима работы ионного источника инжектора осуществлялся на основе данных о 

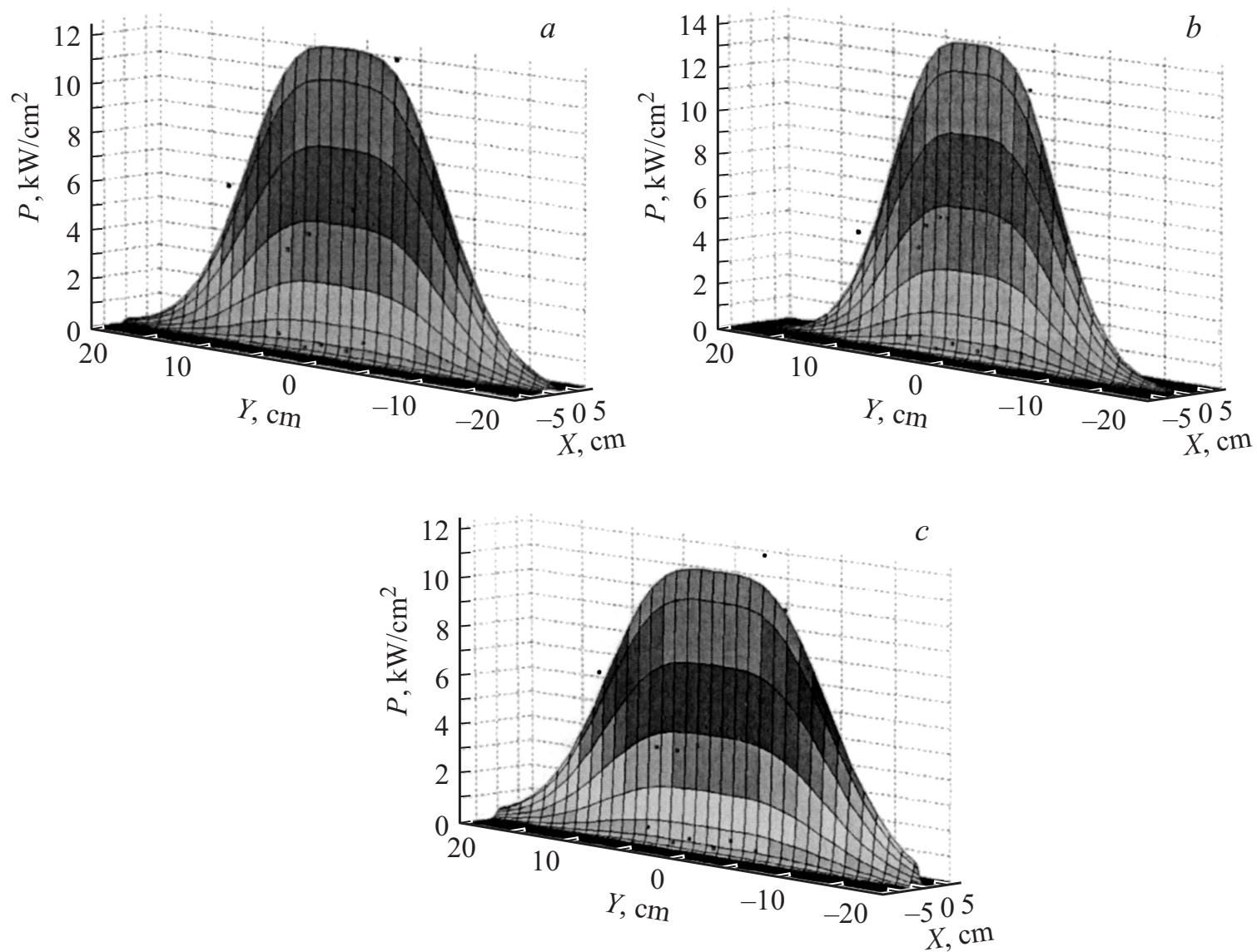

Рис. 7. Реконструкция профиля мощности пучка для разрядов \# 320686 (a), \#320710 (b) и \# 320693 (c) на 90 ms.

профиле мощности атомного пучка, реконструируемом по сигналам с вторично-эмиссионных зондов (22 шт.) приемника пучка. Методика измерений описана в работе [1] и позволяет восстановить профиль распределения плотности потока мощности и, таким образом, оценить качество фокусировки. Для визуализации и анализа данных зондовых измерений использовался код DAS Tools [6].

В начале эксперимента проводилась оценка эффективности нейтрализации по соотношению токовых сигналов с зондов при выключенном $\left(I^{0}+I^{+}\right)$и включенном $\left(I^{0}\right)$ электромагнитном сепараторе [1]. Электромагнит отклоняет неперезарядившиеся ионы из пучка быстрых атомов, направляемого в приемник. Измерения показали, что коэффициент нейтрализации составляет $\sim 0.75$ и соответствует равновесному выходу для бесконечно толстой мишени при заданной энергии водородного пучка, т.е. можно считать, что режим напуска газа в нашем случае не оказывает влияния на эффективность нейтрализации пучка.

Для примера на рис. 6 представлены сигналы с одного из центральных зондов для разных режимов газонапуска при включенном магните (атомный пучок). Видно, что уровень сигнала наиболее постоянен в разряде \# 320710. Результаты реконструкции профилей мощности пучка в
Таблица 3. Плотность мощности на оси, поперечные размеры пучка для различных режимов напуска рабочего газа

\begin{tabular}{c|c|c}
\hline $\mathrm{N}$ & $P, \mathrm{~kW} / \mathrm{cm}^{2}$ & $X \times Y, \mathrm{~cm}$ \\
\hline$\# 320710$ & 14 & $2.16 \times 18.67$ \\
\hline$\# 320686$ & 12 & $2.06 \times 22.87$ \\
\hline$\# 320693$ & 10 & $2 \times 26.1$
\end{tabular}

конце разряда $(t=90 \mathrm{~ms})$, где наиболее заметно влияние режима газонапуска, представлены на рис. 7 и в табл. 3.

Видно, что в разряде \#320710 значение плотности мощности на оси пучка выше, чем в разрядах \# 320686 и \# 320693, а вертикальный размер пучка наименьший. Таким образом, оптимальной является форма управляющего сигнала, примененная в разряде \# 320710.

\section{Заключение}

Для инжектора атомов токамака Глобус-М разработана новая система напуска газа на основе пьезоэлектрического клапана, позволившая регулировать режим работы 
ионного источника в течение импульса. Контроль за работой системы осуществляется с пульта управления инжектором через штатный компьютер. Работоспособность системы была проверена в эксперименте. Для инжектора токамака Глобус-М с источником ионов ИПМ-2 была подобрана оптимальная форма управляющего сигнала для системы подачи газа в ГРК. С учетом имеющегося у новой системы напуска газа запаса по пропускной способности она также может быть использована при работе с ионным источником ИПМ-1, имеющим больший объем ГРК. Для этого потребуется только подобрать оптимальную форму управляющего сигнала.

\section{Благодарности}

Авторы благодарят за частичную финансовую поддержку Минобрнауки России, грант № 14.W01.16.5846МК (Г.С. Курскиев) и РФФИ, грант 16-32-00454-мол_а (П.Б. Щеголев).

\section{Список литературы}

[1] Гусев В.К., Деч А.В., Есипов Л.А. и др. // ЖТФ. 2007. Т. 77. Вып. 9. С. 28-43.

[2] Гусев В.К., Голант В.Е., Гусаков Е.3. и др. // ЖТФ. 1999. Т. 69. Вып. 9. С. 58-62.

[3] Model MV-112. Piezoelectric Gas Leak Valve. Manual. Maxtek. Inc. 17 p.

[4] E14-140; E14-140-M/Manual. M.: L-CARD, 2010. 63 c.

[5] Тельнова A. Неделя науки СПбГПУ: Материалы науч.практич. конф. с междун. участием. Институт физики, нанотехнологий и телекоммуникаций СПбГПУ. Ч. 2. СПб: Изд-во Политехн. ун-та, 2014. $211 \mathrm{c.}$

[6] Игонькина Г.Б., Соколов М.М., Тилинин Г.Н. // Сб. докл. 11-й Всерос. конф. по диагностике плазмы. Троицк, 2005. 